\title{
Ethnomedicinal Studies of Lalmohan Thana in Bhola District, Bangladesh
}

\author{
Sohel MDD ${ }^{1,2^{*}}$, Kawsar MDH $^{2}$, Sumon MDHU $^{3}$ and Sultana $\mathbf{T}^{1,2}$ \\ ${ }^{1}$ Department of Pharmacy, State University of Bangladesh, Dhanmondi, Dhaka-1205, Bangladesh \\ ${ }^{2}$ Incepta Pharmaceuticals Ltd, Dewan Idris Road, Zirabo, Savar, Dhaka, Bangladesh \\ ${ }^{3}$ Department of Pharmacy, University of Asia Pacific, Dhanmondi, Dhaka-1209, Bangladesh
}

*Corresponding author: Sohel MDD, Department of Pharmacy, State University of Bangladesh, Dhanmondi, Dhaka-1205, Bangladesh, Tel: +8801916016974; E-mail: sohelphr15@gmail.com

Rec date: December 22, 2015, Acc date: February 04, 2016, Pub date: February 11, 2016

Copyright: ( 2016 Sohel MDD, et al. This is an open-access article distributed under the terms of the Creative Commons Attribution License, which permits unrestricted use, distribution and reproduction in any medium, provided the original author and source are credited.

\begin{abstract}
The purpose of the present study was to document the medicinal plants of a Unani folk medicinal practitioner in Lalmohan Thana that located in Bhola district in Bangladesh. There is very narrow information about plants used by traditional healers and general people in Bhola District in Bangladesh, for treating general ailments. An ethnomedicinal survey conducted among folk medicinal herbalists of one village in Bhola district resulted in the finding of 146 plants distributed into 64 families used by the herbalists. The various plants were used for treatment of ailments like Urinary Disorders, Contraceptive, Diuretics, Fever, Constipation, Menoxenia, Jaundice, Respiratory Disorders (Coughs, Mucus), Leprosy, Tuberculosis, Sexual disorders, Gastrointestinal Disorders (Dysentery, Diarrhea, Indigestion, Constipation), Vomiting, Helminthiasis, Jaundice, Infections, Heart Disorders, Skin Disorders, Gonorrhea, Urinary Problems, Edema, Typhoid, Liver Disorders, Blood Poisoning, Eye Disorders, Memory Loss, Ovarian Problems, Vaginitis, and Hypertension.
\end{abstract}

This study could play an important role for future phytochemical and pharmacological investigation.

Keywords: Ethno medicinal; Lalmohan Thana; Bhola district; Folk medicine; Bangladesh

\section{Introduction}

In Greece the Unani system of medicine originated. Hippocrates is the father of this system of medicine. Disease is a usually caused by imbalance of the humors. Pharmacotherapy is resorted the balance of the humors. At present, Unani medicine is practiced in the Indian subcontinent countries of India, Bangladesh and Pakistan, the practitioners being known as Hakims. Traditional medicine in Bangladesh is a unique blend of different ethnomedicinal combination [1]. Folk medicinal practitioners (Kavirajes) form the primary healthcare providers to a significant section of the rural and urban population of Bangladesh. They exploit a variety of medicinal plants for treatment of different ailments. Folk medicine possibly is the most common form of these traditional medicinal practices, and folk medicinal practitioners (FMPs) can be found in every village, towns and cities within the country. From ancient time, the tradition of ethno-medicine practice has been established in Bangladesh and such medicine practitioners are known as Kavirajes. According to the WHO, about $80 \%$ of the world's population relies on traditional medicine for their primary health care $[2,3]$. About $80 \%$ of more than 4,000 million inhabitants of the world rely chiefly on traditional medicines for their primary health care needs [4]. Lalmohan is located in Bhola District. Bhola district is an administrative district in southwestern part of Bangladesh, which includes Bhola Island. Bhola Island is the largest island of Bangladesh. The objective of the present study was to document the medicinal plants used by FMPs in one villages of Lalmohan Thana in Bhola district, Bangladesh [5]. Rural people are heavily depend on natural resources due to lack of modern medical knowledge [6]. Thus, over time, a practitioner can build up quite extensive knowledge on the medicinal properties of any given plant species [7]. The development of western medicine is believed to have been influenced by the writing of Greek philosophers, in particular, Hippocrates (460-377 BC) and Aristotle (384-322 BC) [8]. The folk medicinal practitioners do not have their own medicinal books or follow any standardized custom [9]. Medicinal plants play a significant role in the primary healthcare systems for the majority of the rural population. The ethomedicinal knowledge about the use of medicinal plants can be a resource for the scientist to identify potential drugs, thus, proper documentation of this knowledge overtime is very essential to protect them from extinction [10]. It has been estimated that about $64 \%$ of the total global population still remains dependent on traditional medicines for healthcare needs [11].

\section{Materials and Methods}

The survey was carried out in the villages of Purbophara 3No. Ward, Lalmohan Thana, which is located in Bhola district in Bangladesh. It is a part of Lalmohan Thana. The villages had one medicinal practitioner, who practiced folk medicine. However, his name is Hakeem Md. Jamal Uddin (M. M, D.U.M.S) the Hakeem title suggesting that his selection of medicinal plants was influenced by the ancient Unani system of medicine. Actual interviews were conducted with the help of a semistructured questionnaire and the guided field-walk method of Martin and Maundu. Briefly, in this method, the practitioners took the interviewers on guided field-walks through areas from where they collected medicinal plants, pointed out the plants, and as interviewers belonged to the mainstream Bengali-speaking population [12,13]. The interviews were conducted among the locals of different age groups, mostly between 25 to 65 years, including herbal practitioners (Kabiraj). 
Citation: Sohel MDD, Kawsar MDH, Sumon MDHU, Sultana T (2016) Ethnomedicinal Studies of Lalmohan Thana in Bhola District, Bangladesh.

Page 2 of 9

The interviews focused on basic questions concerning the informant's knowledge of the uses of local plants and there different characteristics. A typical question would be: which local plants do you know and/or use? How many people in your area use the plant as medicine? [14]. In this method, the Hakim took the interviewers on guided field-walks through areas from where he collected his medicinal plants, pointed out the plants, and described their uses [15] (Table 1).

\begin{tabular}{|c|c|c|c|c|}
\hline Botanical Name & Local Name & Family & Plant parts used & Uses \\
\hline Abelmoschus esculentus & Bhindi & Malvaceae & Seed & Gastric Disorders \\
\hline Abelmoschus moschatus & Muskdana & Malvaceae & Seed & Tonics, Urinary Discharge \\
\hline Abroma augusta $L$. & Ulatkambal & $\begin{array}{l}\text { Sterculiace } \\
\text { ae }\end{array}$ & Leaf, Root & $\begin{array}{l}\text { Amenorrhoea and Dysmenorrhoea, Regulates Irregular Menses Pain } \\
\text { and Gonorrhea }\end{array}$ \\
\hline Abrus precatorius $L$. & Kunch & $\begin{array}{l}\text { Leguminos } \\
\text { ae }\end{array}$ & Seed, Leaf, Root & Contraceptive, Aphrodisiac \\
\hline Acacia farnesiana (L.) Willd & Belatibabul & Fabaceae & Leaf, Bark & Diuretic, Treat Antiulcer, Anti-Pyritic \\
\hline Acacia nilotica (Linn.) & Babul & $\begin{array}{l}\text { Leguminos } \\
\text { ae }\end{array}$ & Leaf, Gum & Diarrhea, Fever \\
\hline Acalypha indica $L$. & $\begin{array}{l}\text { Muktajhuri, } \\
\text { Biralhatchi }\end{array}$ & $\begin{array}{l}\text { Euphorbiac } \\
\text { eae }\end{array}$ & Leaf & Rheumatism, Constipation, Kill Worms In Sores \\
\hline Acanthus illicifolis $L$. & Harkuch Kanta & $\begin{array}{l}\text { Acanthacea } \\
\mathrm{e}\end{array}$ & Root, Plant & Diuretics \\
\hline Achyranthes Aspera L. & Apang & $\begin{array}{l}\text { Amarantha } \\
\text { ceae }\end{array}$ & Whole Plant & $\begin{array}{l}\text { Prolonged Menstrual Flow, Menoxenia (Abnormal Menses), Habitual } \\
\text { Abortion, Jaundice }\end{array}$ \\
\hline Adhatoda vasica Nees & Basak. & $\begin{array}{l}\text { Acanthacea } \\
\text { e }\end{array}$ & Root, Bark and Leaves & Cough, Asthma \\
\hline Adina cordifolia Benth \& Hook & Kelikadam & Malvaceae & Seed & Kill Worms In Sores \\
\hline Aegle marmelos $L$. & Bael & Rutacae & Fruit, Leaves & Constipation, Cough \\
\hline Albizia lebbeck (L.) Benth. & Sirish & $\begin{array}{l}\text { Leguminos } \\
\text { ae }\end{array}$ & Leaf & Leprosy, Diarrhoea \\
\hline Albizia procera (Roxb.) Benth. & Koroi & $\begin{array}{l}\text { Leguminos } \\
\text { ae }\end{array}$ & Leaf & $\begin{array}{l}\text { The leaves are insecticidal; made into poultice they are applied to } \\
\text { ulcer. }\end{array}$ \\
\hline Allium cepa Linn. & Pyaj & Liliaceae & Bulb & Aphrodisiac, Rheumatism \\
\hline Alocasia Indica (Roxb.) Schott. & Mankachu & Araceae & Roots & Rheumatism, leprosy \\
\hline Aloe Indica Wild. & Ghritakumari & Liliaceae & Skin of Fruit, Leaf & $\begin{array}{l}\text { Digestive problems such as constipation, colitis and irritable bowel } \\
\text { syndrome Kidney stones, Menstrual discomfort }\end{array}$ \\
\hline Alstonia scholaris (L.) R. Br. & Chattim & $\begin{array}{l}\text { Apocynace } \\
\text { ae }\end{array}$ & Bark & Anthelmintic, Antipyretic, Antimalarial \\
\hline Amaranthus spinosus Linn. & Kantanotya & $\begin{array}{l}\text { Amarantha } \\
\text { ceae }\end{array}$ & Leaf, Root & Diuretic, Gonorrhoea \\
\hline Amaranthus tricolor $L$. & Lalshak. & $\begin{array}{l}\text { Amarantha } \\
\text { ceae }\end{array}$ & Leaves & Diuretics \\
\hline Amomum aromaticum Roxb. & Morang elachi & $\begin{array}{l}\text { Zingiberace } \\
\text { ae }\end{array}$ & Fruit & Appetizer \\
\hline $\begin{array}{l}\text { Amorphophallus campanulatus } \\
\text { (Roxb.) Bl. Ex. Decne. }\end{array}$ & Olkachu & Araceae & Tuber & Rheumatism, Abdominal Pain, Elephantiasis \\
\hline Anacardium occidentale $L$. & Cashew Nut & $\begin{array}{l}\text { Anacardiac } \\
\text { eae }\end{array}$ & Fruit & Anti-diabetic, anti-bacterial \\
\hline Ananas Sativus schult. F. & Anaras & $\begin{array}{l}\text { Bromeliace } \\
\text { ae }\end{array}$ & Leaf, fruit & Anthelmintic, diuretic, abortifacient \\
\hline
\end{tabular}


Citation: Sohel MDD, Kawsar MDH, Sumon MDHU, Sultana T (2016) Ethnomedicinal Studies of Lalmohan Thana in Bhola District, Bangladesh. Altern Integr Med 5: 210. doi:10.4172/2327-5162.1000210

Page 3 of 9

\begin{tabular}{|c|c|c|c|c|}
\hline Annona reticulata $L$. & Nona Ata & $\begin{array}{l}\text { Annonacea } \\
\text { e }\end{array}$ & Leaves and seeds & Anthelmintic, Insecticidal \\
\hline Anthocephalus indicus & Kadam & Rubiaceae & Leaf & Anthelmintic \\
\hline $\begin{array}{l}\text { Aphanamixis polystachya (Wall.) } \\
\text { R.N. Parker. }\end{array}$ & Royena & Meliaceae & Bark, Seed Oil & $\begin{array}{l}\text { Spleen and Liver Diseases, Edema, Stimulating Liniment In } \\
\text { Rheumatism }\end{array}$ \\
\hline Arachis hypogaea $L$. & Mata kalai & $\begin{array}{l}\text { Leguminos } \\
\text { ae }\end{array}$ & Fruit & Gonorrhea, Nutritious \\
\hline Areca catechth $L$, & Supari & Palmae & Fruit & Anthelmintic, Rheumatism, Aphrodisiac \\
\hline Argyreia nervosa & Bijarka & $\begin{array}{l}\text { Convolvula } \\
\text { ceae }\end{array}$ & Leaf, Fruit & Rheumatism, Diuretics \\
\hline Aristolochia indica $L$. & Iswarmul. & $\begin{array}{l}\text { Aristolochia } \\
\text { ceae }\end{array}$ & Root, Leaf & Fever, Stimulant \\
\hline Artocarpus heterophyllus Lamk. & Kathal & Moraceae & Fruit & Skin diseases, Nutritious \\
\hline Asparagus racemosus $L$ & Shatamull & Liliaceae & Root & Diuretic, Aphrodisiac, Antidiarrhoeaic \\
\hline Averrhoa carrambola Linn & Kamranga & $\begin{array}{l}\text { Oxalidacea } \\
\mathrm{e}\end{array}$ & Fruit & Antipyretic and anthelmintic, Antioxidant \\
\hline Azadirachta indica A. Juss. & Neem & Meliaceae & Leaf, Bark, Seed Oil & Bacterial, Fungal, Antipyretic and Antimalarial \\
\hline Bacopa moniera L. & Brahmi & $\begin{array}{l}\text { Serophulari } \\
\text { aceae }\end{array}$ & Whole Plant & Reducing anxiety, Improving memory formation \\
\hline $\begin{array}{l}\text { Barringtonia acutangula (L.) } \\
\text { Gaertn. }\end{array}$ & Hijal & $\begin{array}{l}\text { Lacythidace } \\
\text { ae }\end{array}$ & Seed, Bark, Leaf, Root & Diuretics, Fever, Headache \\
\hline Basella alba Linn. & Puishak & $\begin{array}{l}\text { Basellacea } \\
\text { e }\end{array}$ & Leaf & Skin diseases, Sexual weakness, Ulcers and laxative \\
\hline $\begin{array}{l}\text { Benincasa hispida (Thunb.) } \\
\text { Cogn. }\end{array}$ & Chalkumra & $\begin{array}{l}\text { Cucurbitace } \\
\text { ae }\end{array}$ & Seed, Fruit & Epilepsy and Nervous Diseases \\
\hline Blumea lacera (Burm.f.) DC. & Kukurshinga & Asteraceae & Whole plant & Antiviral, Antipyretic \\
\hline Boerhaavia diffusa $L$. & Punarnava & $\begin{array}{l}\text { Nyctaginac } \\
\text { eae }\end{array}$ & Whole plant & Diuretic, Edema \\
\hline Brassica campestris $L$. & Sarisa shak & Cruciferae & Seed & Cough, Leprosy \\
\hline Bombax ceiba $L$. & Shimul-Tula & $\begin{array}{l}\text { Bombacace } \\
\text { ae }\end{array}$ & Root & Increase Sex In Male, Gynecological And Urinogenitial Disorders \\
\hline Buettneria pilosa Roxb. & Harjora & $\begin{array}{l}\text { Sterculiace } \\
\text { ae }\end{array}$ & stem with leaves & treatment of fractured bones \\
\hline Butea monosperma Roxb. & Palas & $\begin{array}{l}\text { Leguminos } \\
\text { ae }\end{array}$ & Seed, Gum & Astringent, anthelmintic \\
\hline Caesalpinia bonduc (L.) Roxb. & Nata & Fabaceae & $\begin{array}{l}\text { Fruit, Root, Bark, } \\
\text { Leaves }\end{array}$ & Antipyretic, Antispasmodic, Anthelmintic, Kidney Troubles, Asthma \\
\hline Cajanus cajan (L.) Huth. & Arhar & Fabaceae & Leaves & Jaundice and Pneumonia \\
\hline Calotropis Gigantea (L.) R. Br. & Akanda & $\begin{array}{l}\text { Asclepiada } \\
\text { ceae }\end{array}$ & Root, Leaf, Bark & Asthma, Emetic, Leprosy, Rheumatism \\
\hline Capsicum annuum $L$ & Jhal marich & Solanaceae & Fruit & Carminative, Stimulant, Sores, Tonic \\
\hline Cerica papaya Linn & Papay & Caricaceae & Fruit & Carminative, Eczema, Warts, Anthelmintic, Digestion problems \\
\hline Carum roxbunghianum Benth. & Radhuni & Apiaceae & Seed & abdominal spasm (colic) \\
\hline Cassia alata (L.) Gaertn. & Daud pata & $\begin{array}{l}\text { Leguminos } \\
\text { ae }\end{array}$ & Leaf & Skin diseases, Microbial infections. \\
\hline
\end{tabular}


Citation: Sohel MDD, Kawsar MDH, Sumon MDHU, Sultana T (2016) Ethnomedicinal Studies of Lalmohan Thana in Bhola District, Bangladesh. Altern Integr Med 5: 210. doi:10.4172/2327-5162.1000210

Page 4 of 9

\begin{tabular}{|c|c|c|c|c|}
\hline Cassia fistula $L$. & Sonalu & $\begin{array}{l}\text { Leguminos } \\
\text { ae }\end{array}$ & Leaf, Bark, Root, Fruit & Helminthiasis, Constipation, Rheumatism \\
\hline Cassia occidentalis Linn. & Kasondi & $\begin{array}{l}\text { Caesalpinia } \\
\text { ceae }\end{array}$ & Leaf, Seed & Hepatotoxicity \\
\hline Cassia tora Linn. & Chakunda & $\begin{array}{l}\text { Caesalpinia } \\
\text { ceae }\end{array}$ & Seed, Fruit & Skin diseases like ringworm and itching, Anthelmintic \\
\hline Catharanthus roseus $L$. & Nyantara & $\begin{array}{l}\text { Apocynace } \\
\text { ae }\end{array}$ & Root, Leaf & Diabetes mellitus, Hypotensive \\
\hline $\begin{array}{l}\text { Cayratia pedata (Lam.) Juss. Ex } \\
\text { Gagnep. }\end{array}$ & Goalilata & Vitaceae & Leaf & Astringent \\
\hline Centella asiatica $L$. & Thankuni & $\begin{array}{l}\text { Umbellifera } \\
\text { e }\end{array}$ & Whole Plant, leaf & Dysentery, Stomach pain, Memory tonic, Diuretic \\
\hline Chenopodium album $L$. & Bathu sag & $\begin{array}{l}\text { Chenopodi } \\
\text { aceae) }\end{array}$ & Seed, plant & Improves the appetite, Abdominal pain \\
\hline $\begin{array}{l}\text { Chrysopogon } \quad \text { aciculatus } \\
\text { (Retz.)Trin }\end{array}$ & Premkata & Poaceae & Root, Seed & Anthelmintic \\
\hline Cinnamomum tamals Nees & Tejpata & Lauraceae & Leaf & Heart disease, Gastrointestinal disorders, Diarrhea \\
\hline Citrullus lanatus (Thunb.) Mats. & Tarmuj & $\begin{array}{l}\text { Cucurbitace } \\
\text { ae }\end{array}$ & Seed, Rife fruit & Cooling, Refreshing and stomachic, Laxative, Diuretic, Tonic \\
\hline $\begin{array}{l}\text { Citrus aurantifolia (Christm.) } \\
\text { Swingle }\end{array}$ & Lebu, Kaghzilebu & Rutaceae & Fruit, Leaves & Appetizer, Eczema \\
\hline Cleome viscosa $L$. & Hurhuria & $\begin{array}{l}\text { Capparidac } \\
\text { eae }\end{array}$ & Seed & Stomachic, laxative, Diuretic \\
\hline Clerodendrum indicum $L$. & $\begin{array}{l}\text { Bamanhati, } \\
\text { Banchat, }\end{array}$ & $\begin{array}{l}\text { Verbenacea } \\
\text { e }\end{array}$ & Root, Leaf, & $\begin{array}{l}\text { Respiratory problems, Cough, Irregular menstruation, Irregular blood } \\
\text { pressure }\end{array}$ \\
\hline Clerodendrum viscosum Vent. & Vant, Ghetu & $\begin{array}{l}\text { Verbenacea } \\
\text { e }\end{array}$ & Leaf, Root, Fruit & Scabies, Fever, Anthelmintic, \\
\hline Coccinia grandis (L.) Voigt & Telakucha & $\begin{array}{l}\text { Cucurbitace } \\
\text { ae }\end{array}$ & Leaf & Hypertension, Diabetes, Jaundice \\
\hline Cocos nucifera $L$. & Narikel & Arecaceae & Leaf, Fruit & Diuretics, Keep head cool, diabetes. \\
\hline Corchorus capsularis $L$. & Pat shakh & Tiliaceae & Leaf, Seed & Dysentry \\
\hline Coriandrum Sativum Linn. & Dhonia & Apiaceae & Seed & Loss of appetite \\
\hline Crataeva nurvala Buch-Ham & Barun & $\begin{array}{l}\text { Capparidac } \\
\text { eae }\end{array}$ & Leaf, Root, Bark & To promote appetite and decrease secretion of bile \\
\hline Cucumis sativus $L$. & Khira & $\begin{array}{l}\text { Cucurbitace } \\
\text { ae }\end{array}$ & Fruit, Seed & Reduce cholesterol, Diuretics \\
\hline Cucurbita maxima Duch. & Calakumro & $\begin{array}{l}\text { Cucurbitace } \\
\text { ae }\end{array}$ & Seed & Diuretics, Anthelmintic \\
\hline Cuminum cyminum Linn & Jeera & $\begin{array}{l}\text { Umbellifera } \\
\text { e }\end{array}$ & Fruit & Astringent, Carminative \\
\hline Cucuma longa $L$. & Kacha Holud & $\begin{array}{l}\text { Zingiberace } \\
\text { ae }\end{array}$ & Rhizomes & Allergy, Inflammation, Skin disease, Anthelmintic \\
\hline Cuscuta reflexa Roxb. & Swarnalata & $\begin{array}{l}\text { Cuscutacea } \\
\text { e }\end{array}$ & Seed, Stem & Flatulence, stomach pain, constipation \\
\hline Cymbopogon citratus (Dc.) Stapf. & $\begin{array}{l}\text { Lebugandhi } \\
\text { Ghas. }\end{array}$ & Graminae & leaves & Fever, Rheumatism, appetizer and Anthelmintic \\
\hline Cyperus rotundus Linn. & Mutha Ghas & $\begin{array}{l}\text { Cyperacea } \\
\text { e }\end{array}$ & Rhizomes & Stimulant, Stomachic aromatic \\
\hline
\end{tabular}


Citation: Sohel MDD, Kawsar MDH, Sumon MDHU, Sultana T (2016) Ethnomedicinal Studies of Lalmohan Thana in Bhola District, Bangladesh. Altern Integr Med 5: 210. doi:10.4172/2327-5162.1000210

Page 5 of 9

\begin{tabular}{|c|c|c|c|c|}
\hline Dalbergia bissoo Roxb. & Shishu & $\begin{array}{l}\text { Leguminos } \\
\text { ae }\end{array}$ & Leaf, bark, seed & Leprosy, scabies, Astringent \\
\hline Datura meta Linn. & Dhutura & Solanaceae & Leaf, Root, Seed & Asthma, Rheumatism, Fever, pain \\
\hline Derris trifoliata Lour. & Panlata & Fabaceae & Bark, Stem & Stimulant, Insecticides, Rheumatism \\
\hline Dillenia indica Linn & Chalta & $\begin{array}{l}\text { Dilleniacea } \\
\text { e }\end{array}$ & Fruit, Leaves & tonic and laxative; used in diarrhoea \\
\hline Diospyros peregrine Gurke & Desi gabh & Ebenaceae & Fruit & Dysentery and diarrhea, Diuretics \\
\hline Dioscorea bulbifera $L$. & Pagla Alu & $\begin{array}{l}\text { Dioscoreac } \\
\text { eae }\end{array}$ & tubers & dysentery, diarrhea, stomachic \\
\hline Dolichos lablab linn. & Shim & $\begin{array}{l}\text { Leguminos } \\
\text { ae }\end{array}$ & Seed, Leaf & Astringent, Nausea, vomiting and abdominal pains \\
\hline Eclipta alba Hassk & Kesuriya & Asteraceae & Whole Plant & $\begin{array}{l}\text { Protect the liver, tonic, deobstruent in hepatic and splenic } \\
\text { enlargements, jaundice }\end{array}$ \\
\hline Elaeocarpus robustus linn. & Jalpai, Jalphui. & $\begin{array}{l}\text { Elaeocarpa } \\
\text { ceae }\end{array}$ & Fruit, Leaf & Splenic enlargements, Lethargy to food \\
\hline Elettaria cardamomum (L.) Maton & Elas & $\begin{array}{l}\text { Zingiberace } \\
\text { ae }\end{array}$ & Fruit & $\begin{array}{l}\text { Remedy for impotence and low sexual response. abdominal pains, } \\
\text { Appetizer }\end{array}$ \\
\hline Embelia ribes Burm F & Biranga & $\begin{array}{l}\text { Myrsinacea } \\
\text { e }\end{array}$ & Seed & Anthelmintic \\
\hline Eryngium foetidum $L$. & Bon dhonia & Apiaceae & Root, Leaf & Diuretics, colds, coughs, hypertension, Arithitis \\
\hline Erythrina variegate $L$ & Madar & Fabaceae & Leaf, Bark, Root & Fever, Anthelmintic, \\
\hline Eucalyptus citriodora Hook. & Eucalyptus & Myrtaceae & Juice of leaves & $\begin{array}{l}\text { anti-inflammatory and analgesic qualities and can be applied to } \\
\text { wounds to help prevent infection }\end{array}$ \\
\hline Eugenia jambolana (Lam) & Jum & Myrtaceae & Juice of young leaves & Dysentery and diarrhoea. \\
\hline Eupatorium odoratum Linn & Japanilata & Compositae & Leaf & The juice of the leaf is applied on wounds to cheek Bleeding. \\
\hline Ficus benghalensis $L$. & Bot & Moraceae & $\begin{array}{l}\text { Bark, Gum, leaf, fruit, } \\
\text { root }\end{array}$ & Gonorrhea, Venereal diseases, Abscess, astringent, aphrodisiac \\
\hline Ficus glomerata Roxb. & Jagadumur & Moraceae & Fruit, Bark & $\begin{array}{l}\text { Leucorrhoea, biliousness, burning sensation, fatigue, Diabetes, } \\
\text { Dysentry, nose bleeding }\end{array}$ \\
\hline Ficus hispida Linn. & Kakdumul & Moraceae & Bark, fruit, leaf, root & Galactagogue, emetic, anaemia, haemorrhoids \\
\hline Glinus oppositifolius $L$. & Gima shak & $\begin{array}{l}\text { Molluginace } \\
\text { ae }\end{array}$ & Whole plant & Abdominal pain and jaundice, loss of appetite, indigestion \\
\hline $\begin{array}{l}\text { Glycosmis pentaphylla (Retz.) A. } \\
\text { Dc. }\end{array}$ & $\begin{array}{l}\text { Ashshaora, } \\
\text { Datmajan, }\end{array}$ & Rufaceae & $\begin{array}{l}\text { Root, Leaf, Stem, Fruit, } \\
\text { Whole Plant, }\end{array}$ & $\begin{array}{l}\text { Cough, Rheumatism, Anaemia And Jaundice, Eczema, Pimple, } \\
\text { Rheumatism, Dysentery, Dental Caries }\end{array}$ \\
\hline Gmelina arborea $L$. & Gamar & $\begin{array}{l}\text { Verbenacea } \\
\text { e }\end{array}$ & Bark, Root & Astringent, Tonic \\
\hline Hedyotis corymbosa (L.) Link. & Khetpapra & Rubiaceae & Whole Plant & Jaundice, Liver Disease, Fever, Heat Eruption, \\
\hline $\begin{array}{l}\text { Hygrophila auriculata (Schum.) } \\
\text { Heyne. }\end{array}$ & $\begin{array}{l}\text { Kulekhara, } \\
\text { Talmakhna. }\end{array}$ & $\begin{array}{l}\text { Acanthacea } \\
\mathrm{e}\end{array}$ & Whole Plant & Diuretics, Jaundice, Gonorrhea, Urinary Discharges, Inflammations \\
\hline Imperata cylindrica Rausch. & Ulu & Poaceae & Roots & Fever \\
\hline Ipomea aquatica Forsk. & $\begin{array}{l}\text { Kalmi, } \quad \text { Kalmi } \\
\text { Shak. }\end{array}$ & $\begin{array}{l}\text { Convolvula } \\
\text { ceae }\end{array}$ & Root, leaves & Plants are used in leucoderma, biliousness, carminative \\
\hline Ipomoea batatus (L.) Lamk. & Misti Alu & $\begin{array}{l}\text { Convolvula } \\
\text { ceae }\end{array}$ & Tubers, Root & Nutritional Source, Diarrhoea \\
\hline Ipomoea mauritiana Jacq. & Bhuikumra & $\begin{array}{l}\text { Convolvula } \\
\text { ceae }\end{array}$ & Root, Tubers & Sexual Disabilities, Galactagogue \\
\hline
\end{tabular}


Citation: Sohel MDD, Kawsar MDH, Sumon MDHU, Sultana T (2016) Ethnomedicinal Studies of Lalmohan Thana in Bhola District, Bangladesh. Altern Integr Med 5: 210. doi:10.4172/2327-5162.1000210

Page 6 of 9

\begin{tabular}{|c|c|c|c|c|}
\hline Jatropha curcus Linn. & $\begin{array}{l}\text { Bagh Verenda, } \\
\text { Ban Verenda }\end{array}$ & $\begin{array}{l}\text { Euphorbiac } \\
\text { eae }\end{array}$ & Seed, Leaf & Purgative \\
\hline Kalanchoe pinnata (Lam.)Pers. & Patharkuchi; & $\begin{array}{l}\text { Crassulace } \\
\text { ae }\end{array}$ & Leaves & Diuretic \\
\hline Lagenaria siceraria (Mol.) Stan. & Lau, Kadu & $\begin{array}{l}\text { Cucurbitace } \\
\text { ae }\end{array}$ & Fruit and seed & Diuretic, headache \\
\hline $\begin{array}{l}\text { Lannea Coromandelica (Houtt.) } \\
\text { Merr. }\end{array}$ & Kamila & $\begin{array}{l}\text { Anacardiac } \\
\text { eae }\end{array}$ & Bark & Astringent, Jaundice \\
\hline Lawsonia inermis Linn. & Mehedi, Mendi, & Lythraceae & Leaf, Bark & Arthritis, Skin disease, \\
\hline LENS culinaris Medik. & Musuri & Fabaceae & Seeds & Constipation \\
\hline Lippia nodiflora (L.) Rich. & Bhui Okar & $\begin{array}{l}\text { Verbenacea } \\
\text { e }\end{array}$ & Leaf, Plant & $\begin{array}{l}\text { diuretic, stomachic, good for ulcers, wounds, asthma, bronchitis, } \\
\text { diarrhoea }\end{array}$ \\
\hline Ludwigia Adscendens (L.) Hara. & Kesardam & $\begin{array}{l}\text { Onagracea } \\
\mathrm{e}\end{array}$ & leaf & Dysentery \\
\hline Lycopersicon lycopersicum (L.) & $\begin{array}{l}\text { Tomato, bilati } \\
\text { beguna }\end{array}$ & Solanaceae & Fruit & Antioxidant \\
\hline Mangifera indica $L$. & Aam & $\begin{array}{l}\text { Anacardiac } \\
\text { eae }\end{array}$ & Leaf, Fruit, Seed, & Toothache, Astringent, Diuretics \\
\hline Manilkara achras (Mill.) Fosberg & Sofeda & Sapotaceae & Seed & Fever, tonic, diuretica \\
\hline Marsilea quadrifolia Linn. & Sushni saag & $\begin{array}{l}\text { Marseliace } \\
\text { ae }\end{array}$ & Leaf & Hypnotic \\
\hline Melia sempervirens $L$. & Ghoranim & Meliaceae & Bark & Diuretics, leprosy, Anthelmintic \\
\hline Mentha spicata Linn. & Pudina & Labiatae & Whole plant & Abdomina; pain, Constipation \\
\hline Mimosa pudica Linn. & 'Lojjaboti & Fabaceae & Whole plant & Diuretics, uterine complaints, inflammation, fatigue \\
\hline Momordica charantia Linn. & Korolla & $\begin{array}{l}\text { Cucurbitace } \\
\text { ae }\end{array}$ & leaf & Diabetes, helminthiasis, Ulcer \\
\hline $\begin{array}{l}\text { Momordica cochinchinensis } \\
\text { (Lour) Spreng. }\end{array}$ & Kakroal & $\begin{array}{l}\text { Cucurbitace } \\
\text { ae }\end{array}$ & Fruit & Diabetes, Abdominal pain, Stimulent \\
\hline Musa paradisiacal L. & Kala & Musaceae & Root, tubers, Fruit & Dysentery, Astringent, BP \\
\hline Nerium indicum Mill. & Korobi & $\begin{array}{l}\text { Apocynace } \\
\text { ae }\end{array}$ & Root, leaves & Skin disease, Leprosy \\
\hline Nigella sativa Linn. & Kalojira, Kalijira & $\begin{array}{l}\text { Ranunculac } \\
\text { eae }\end{array}$ & Seed & Stimulant and diuretic. \\
\hline Nymphaea nouchali Burm.f & Sapla & $\begin{array}{l}\text { Nymphaeac } \\
\text { eae }\end{array}$ & Flower & Dysentery, Diarrhea, Heart disease, \\
\hline Ocimum basilicum Linn. & Babui Tulshi & Lamiaceae & Whole plant & Fever, carminative, \\
\hline Ocimum sanctum Linn. & Tulshi & Lamiaceae & Leaf & Fever, cough \\
\hline Phoenix Sylvestris (L.) & Khejur & Arecaceae & Fruit, Root & Toothache, Nutritious \\
\hline Phyllanthus emblica L. & Amloki & $\begin{array}{l}\text { Euphorbiac } \\
\text { eae }\end{array}$ & Fruit & Vomiting, cough, indigestion, jaundice, Skin disease \\
\hline Piper nigrum $L$. & Gol morich & piperaceae & Seed, Fruit & Chest and joint pain, hair loss, diuretics \\
\hline Piper betle $L$. & Paan pata & piperaceae & leaf & Brest and prostate cancer, stomach disorder \\
\hline Psidium guajava Linn, & Piyara & Myrtaceae & leaves & Scarby, menstrual problem, diarrhea \\
\hline Punica granatum $L$. & Dalim & Punicaceae & Bark & Anaemia, anthelmintics \\
\hline
\end{tabular}


Citation: Sohel MDD, Kawsar MDH, Sumon MDHU, Sultana T (2016) Ethnomedicinal Studies of Lalmohan Thana in Bhola District, Bangladesh.

Page 7 of 9

\begin{tabular}{|c|c|c|c|c|}
\hline Raphanus Sativus Linn & Mula & Cruciferae & Leaf, Seed, Root & Carminative, stimulant, increase digestion \\
\hline $\begin{array}{l}\text { Rauwolfia serpentina } \\
\text { (L.) Benth. ex Kurz }\end{array}$ & Sarpogondha & $\begin{array}{l}\text { Apocynace } \\
\text { ae }\end{array}$ & Root & Root extract is directly feed to neutralize the snake venom \\
\hline Rosa damascena mill $L$ & Golap & Rosaceae & Flower & Carminative, Astringent, Tonic, Vaginal disease \\
\hline Rumex Maritimus L & Ban Palang & $\begin{array}{l}\text { Polygonace } \\
\text { ae }\end{array}$ & Plant & Refrigerant \\
\hline Rumex vesicarius Linn. & Chuka Sak & $\begin{array}{l}\text { Polygonace } \\
\text { ae }\end{array}$ & Leaf & Refrigerent, diuretics, Appetizer \\
\hline Sesanum indicum $L$. & Til & $\begin{array}{l}\text { Pedaliacea } \\
\mathrm{e}\end{array}$ & Leaf, Seed, oil & Dysentery \\
\hline Solanum melongena $L$. & Begun & Solanaceae & Leaf, Seed, Fruit & Laxative, Cough \\
\hline Tamarindus indica Linn & Tentul & $\begin{array}{l}\text { Leguminos } \\
\text { ae }\end{array}$ & Seed & Constipation \\
\hline Terminalia arjuna & Arjun & $\begin{array}{l}\text { Combretac } \\
\text { eae }\end{array}$ & Bark & Heart disease \\
\hline Zingiber officinale Rose. & Ada & $\begin{array}{l}\text { Zingiberace } \\
\text { ae }\end{array}$ & Rhizomes & stomachic, appetiser, expectorant \\
\hline Zizyphus mauritiana Lamk. & Boroi & $\begin{array}{l}\text { Rhamnace } \\
\text { ae }\end{array}$ & Fruit, Root & astringent, laxative, stomachic \\
\hline
\end{tabular}

Table 1: Ethnomedicinal usesof medicinal plants in Lalmohan Thana, Bhola district.

\section{Results and Discussion}

In our study, we found large number participants who are female. According to Ikhtiar Alam SM, in Developing countries, society is, in general, male dominated in terms of participation in household decision making [16]. In Bangladesh, the male villagers are more knowledgeable than female in term of medicinal knowledge. Again, aged person are more knowledgeable than younger one.

According to Dr. Abdul Ghani almost 455 medicinal plants name so far been enlisted as growing or available in Bangladesh [17] (Figure 1).

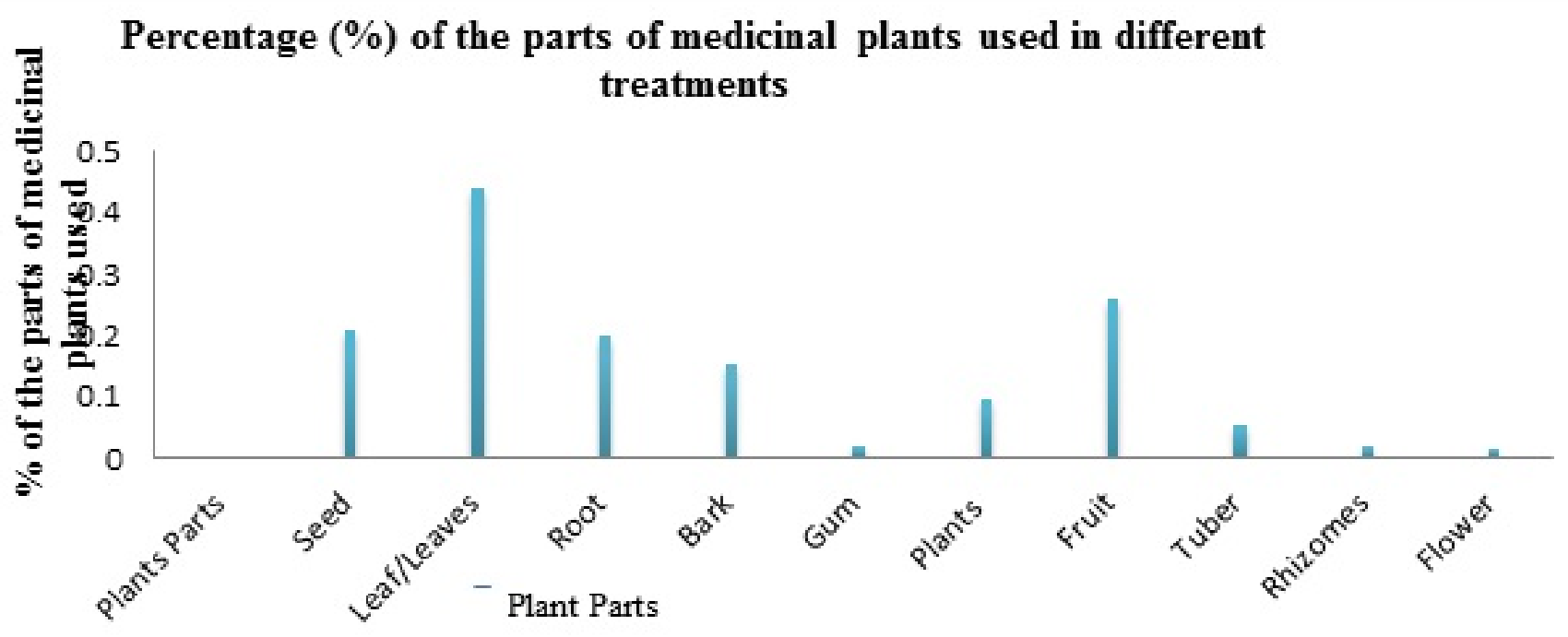

Figure 1: Parts of medicinal plants used in different preparations in the study area.

The Hakim was observed to use a total of almost 146 plants distributed into 64 families for different ailments. The percentage of medicinal plants parts Leaves (43.83\%), Seed (20.55\%), Root (19.86\%), Bark (15.07\%), Gum (2.05\%), Plants (9.59\%), Fruit (26.03\%), Tuber 
Citation: Sohel MDD, Kawsar MDH, Sumon MDHU, Sultana T (2016) Ethnomedicinal Studies of Lalmohan Thana in Bhola District, Bangladesh.

Page 8 of 9

(5.48\%), Rhizomes (2.05\%), Flower (1.37\%) are used for treatment of different ailments. The various plants were used for treatment of ailments like Urinary Disorders, Contraceptive, Diuretics, Fever, Constipation, Menoxenia, Jaundice, Respiratory Disorders (Coughs, Mucus), Leprosy, Tuberculosis, Sexual disorders, Gastrointestinal Disorders (Dysentery, Diarrhea, Indigestion, Constipation), Vomiting, Helminthiasis, Jaundice, Infections, Heart Disorders, Skin Disorders, Gonorrhea, Urinary Problems, Edema, Typhoid, Liver Disorders, Blood Poisoning, Eye Disorders, Memory Loss, Ovarian Problems, Vaginitis, and Hypertension (Figure 1).
Percentages were calculated as the ratio between the number of plants in which a certain part is used and the total number of plants.

\section{Data analysis}

The FC of the species of plants being utilized was evaluated using the formula: $\mathrm{FC}=$ (Number of times a particular species was mentioned/Total number of times that all species were mentioned) $\mathrm{x}$ 100 [18] (Figure 2).

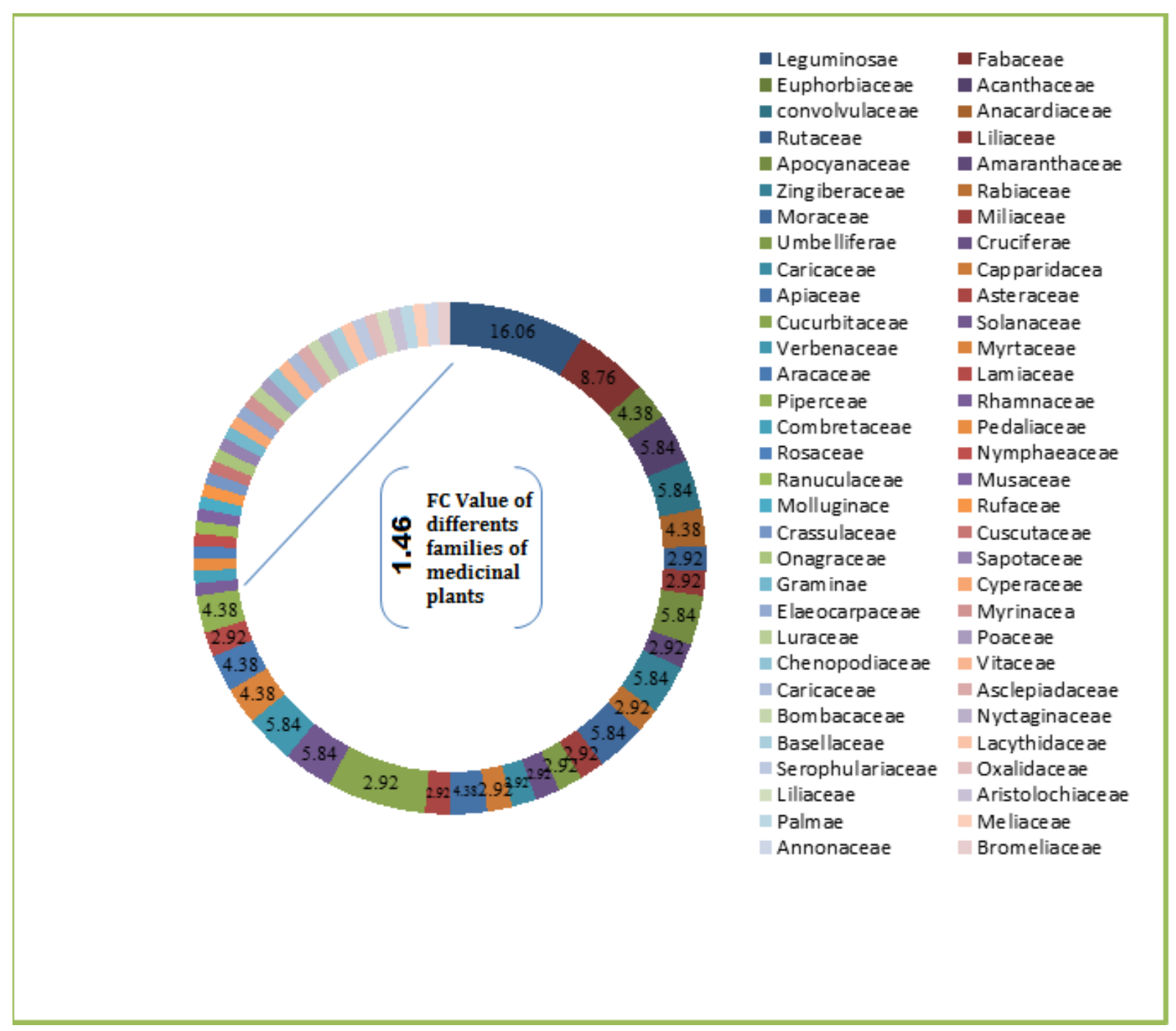

Figure 2: FC Value of different families of medicinal plants.

\section{Conclusion}

Our study reveals that plants are still a major source of medicine for the local communities of most of the portions of our surveyed area, as modern health care facilities are still not sufficient. This report may represents a useful and long-lasting document, which can contribute to preserve knowledge on the use of medicinal plants in this region and also stimulate the interest of future generations on traditional healing practices. The information provided in the paper is limited and there is a scope to initiate further ethno botanical study among the communities to gather information as far as possible. The medicated claims incorporated in the study need to be evaluated through phytochemical and pharmacological investigations to discover their potentiality as drugs. It is urgent need for documenting these before such valuable knowledge becomes inaccessible and extinct. 
Citation: Sohel MDD, Kawsar MDH, Sumon MDHU, Sultana T (2016) Ethnomedicinal Studies of Lalmohan Thana in Bhola District, Bangladesh. Altern Integr Med 5: 210. doi:10.4172/2327-5162.1000210

Page 9 of 9

\section{Acknowledgments}

Authors would like to express gratitude and thanks to Traditional Health Practitioners and people involved in the interviews for providing information about the medicinal applications of the plants. The authors are also grateful to the Department of Pharmacy, State University of Bangladersh, for their suggestion and information during this research.

\section{References}

1. Ocvirk S, Kistler M, Khan S, Talukder SH, Hauner H, et al. (2013) Traditional Medicinal Plants Used For The Treatment of Diabetes in Rural and Urban Areas of Dhaka, Bangladesh, An Ethnobotanical Survey. Journal of Ethnobiology and Ethnomedicine 9: 1-8.

2. Behera KK (2006) Ethnomedicinal plants used by the tribals of Similipal Bioreserve Orissa, India: a pilot studies. Ethnobotanical Leaflets 10:149-173.

3. Khisha T, Karim R, Chowdhury SR, Banoo R (2012) Ethnomedical Studies of Chakma Communities of Chittagong Hill Tracts, Bangladesh. Bangladesh Pharmaceutical Journal 15: 59-67.

4. Tumpa SI, Hossain Md. I, Ishika T (2014) Ethnomedicinal use of herbs by indigenous medicine practitioners of Jhenaidah district, Bangladesh. Journal of Pharmacognosy and Phytochemistry 3:23-33.

5. Afrin M, Rukaiya U, Sharmin S, Jannat K, Akter M, et al. (2015) Ethnomedicinal plants of three folk medicinal practitioners in two villages of Khulna district, Bangladesh. Journal of Chemical and Pharmaceutical Research 7:220-225.

6. Tariq A, Mussarat S, Adnan M, Abd_Allah EF, Hashem A, et al. (2015) Ethnomedicinal Evaluation of Medicinal Plants Used against Gastrointestinal Complaints. BioMed Research International Article ID 892947, 14.

7. Tuhin Md. IH, Asaduzzaman Md., Islam E, Khatun Z, Rahmatullah M (2013) Medicinal plants used by folk medicinal herbalists in seven villages of Bhola district, Bangladesh. American-Eurasian Journal of Sustainable Agriculture 7: 210-218.
8. Chikezie PC, Ojiako OA (2015) Herbal Medicine: Yesterday, Today and Tomorrow Altern Integr Med 4:1-5.

9. Rahmatullah M, Mollik Md. AH, Paul AK, Jahan R, Khatun Mst. A, et al. (2010)A Comparative Analysis of Medicinal Plants used to treat Gastrointestinal Disorders in two sub-districts of Great Khulna Division, Bangladesh. Advances in Natural and Applied Sciences 4: 22-28.

10. Bhowmik R, Saha MR, Rahman Md. A, Islam Md. Anwar Ul (2014) Ethnomedicinal Survey of Plants in the Southern District Noakhali, Bangladesh. Bangldesh Pharmaceutical Journal 17: 205-214.

11. Rahmatullah M, Mollik AH, Ali M, Abbas FB, Jahan R, et al. (2011) An Ethnomedicinal Survey of Vitbilia Village in Sujanagar Sub-District of Pabna District, Bangladesh. American-Eurasian J. Agric \& Environ Sci 10: 106-111.

12. Martin GJ (1995) Ethnobotany: A 'People and Plants' Conservation Manual, Chapman and Hall, London; page 268.

13. Maundu P (1995).Methodology for collecting and sharing indigenous knowledge: a case study. Indigenous Knowledge and Development Monitor 3: 3-5.

14. Sajib NH, Uddin, SB (2015) Ethnomedicinal Study of Plants in Hathazari, Chittagong, Bangladesh. Pertanika J Trop Agric Sci 38: 197-210.

15. Muttaki AA, Ahmed Z, Islam Md. S, Opu SA, Sonda MK, et al. (2014) Medicinal plants and formulations of a Unani folk medicinal practitioner of Bhola district, Bangladesh. Journal of Chemical and Pharmaceutical Research 6:231-238.

16. Alam SMI (1998) Role of Women in Decision Making and Economic Contribution at Household Level. Journal of International Affairs 4: 13-15.

17. Ghani A (1998) Medicinal Plants: Chemical Constituents and Uses of the Medicinal Plants of Bangladesh.

18. Dey AK, Mamun OR, Rashid M, Millat MS, Rashid MM (2014) Ethnobotanical survey of medicinal plants used by traditional health practitioners and indigenous people in different districts of Chittagong division, Bangladesh. International Journal of Pharmaceutical Science 3: 01-07. 